\title{
Estudio epidemiológico de un brote de casos con síntomas irritativos en trabajadores del laboratorio de microbiología y análisis clínicos en un hospital público de tercer nivel
}

Outbreak study of workers with irritative symptoms woking in the microbiology and clinical analysis laboratories of a tertiary public hospital

José María Ramada Rodilla1,2,3

Ana Beltran Fonollosa ${ }^{1}$

Consol Serra, ${ }^{1,2,3}$

Fernando G. Benavides ${ }^{1,2,3}$

'Centro de investigación en Salud Laboral-Universidad Pompeu Fabra (CiSAL-UPF),

Barcelona, España.

2Institut Hospital del Mar d'Investigacions Mèdiques (IMIM), Barcelona, España.

${ }^{3}$ CIBER de Epidemiología y Salud Pública (CIBERESP), Madrid, España.

Fechas · Dates

Recibido: 2019.12.10

Aceptado: 2020.04.28

Publicado: 2020.07.15

\section{Correspondencia $\cdot$ Corresponding Author}

José María Ramada Rodilla

JRamada@parcdesalutmar.cat 


\section{Resumen}

Antecedentes: En abril de 2018 se detectó un número inusualmente elevado de síntomas irritativos de ojos, garganta y nariz, síntomas inespecíficos digestivos y mareos en trabajadores de los laboratorios de microbiología (LAM) y análisis clínicos (LAC) de un hospital de tercer nivel de Mallorca, asociados a olores también inhabituales.

Objetivo: Confirmar la existencia del brote, identificar sus causas, y contribuir a su control y prevención.

Métodos: Estudio epidemiológico del brote con componentes cualitativo y cuantitativo. El primero se basó en la técnica de Grupos Focales. El estudio cuantitativo permitió elaborar las curvas epidémicas, en base a las visitas de los trabajadores por molestias al Servicio de Salud Laboral (SSL) y los episodios de incapacidad temporal (IT). Finalmente, se revisaron los informes higiénicos disponibles, valorando las posibles relaciones con la distribución de los casos.

Resultados: Mediante los grupos focales se establecieron dos definiciones operativas de caso, junto a la identificación de las posibles explicaciones del brote según las percepciones de los trabajadores. El estudio cuantitativo confirmó la existencia de un brote en el LAM, descartándolo en el LAC. Los episodios de IT en el LAM entre las semanas 16 y 39 excedieron en cuatro veces el número de casos esperados. El número de visitas al SSL también mostró un exceso de casos en las mismas semanas.

Conclusiones: El estudio cuantitativo confirma la existencia de un brote epidémico que ha finalizado, si bien el estudio cualitativo pone de manifiesto la continuación de los malos olores y molestias. La mejora de los sistemas de vigilancia epidemiológica facilitará la monitorización y control de otros posibles brotes en el futuro.

Palabras clave: Brote, Brote epidémico, Curvas epidémicas, Síntomas irritativos, Grupos focales.

\section{Abstract}

Background: In April 2018, an unusually high number of eye, throat and nose irritative symptoms, nonspecific gastrointestinal symptoms and dizziness were identified in workers in the microbiology (LAM) and clinical analysis (LCA) laboratories of a tertiary public hospital in Mallorca; these symptoms were also associated with perception of unusual odors.

Objective: To confirm the presence of an outbreak, identify its causes, and contribute to its prevention and control.

Methods: Epidemiological study of the outbreak involving qualitative and quantitative methods. The qualitative component was based on conducting focus groups The quantitative study allowed us to develop epidemic curves, based on employee visits to the hospital's occupational health service (OHS) due to either symptoms or episodes of sickness absence (SA). Lastly, available industrial hygiene reports were reviewed to examine possible relationships with the distribution of cases.

Results: Two operational case definitions were established through the focus groups, along with identification of possible explanations for the outbreak based on employee perception. The quantitative study confirmed the outbreak in the LAM, but not in the LCA. The SA epi- 
sodes in the LAM between weeks 16 and 39 exceeded the number of expected cases fourfold. We also detected an excess of visits to the OHS during the same time period.

Conclusions: The quantitative study confirmed an epidemic outbreak that has since ended, although the qualitative study indicated the persistence of bad smell and discomfort. The improvement of epidemiological surveillance systems as a result of the study will facilitate the monitoring and control of future possible outbreaks.

Keywords: Outbreak, epidemic outbreak, epidemic curves, irritative symptoms, focus groups

\section{Introducción}

El término "brote epidémico" hace referencia a un aumento inusual del número de casos de una determinada enfermedad en una población específica en un periodo de tiempo determinado. Tal como lo define la OMS, es la aparición de casos de enfermedad que exceden la expectativa normal(1).

La mayoría de los brotes de problemas de salud se debe a causas infecciosas (brotes de enfermedades transmisibles), aunque también pueden existir brotes producidos por alimentos, toxinas y otros agentes químicos ${ }^{(2,3)}$.

En la VII Encuesta Nacional de Condiciones de Trabajo, se estima que el 23\% de los trabajadores están expuestos a agentes químicos y, entre el personal sanitario, el porcentaje de expuestos asciende al 34\%(4). Estos datos son muy similares a nivel europeo, ya que en la $6^{\mathrm{a}}$ Encuesta Europea de Condiciones de Trabajo se estima que el $21 \%$ de los trabajadores manipula productos o sustancias químicas, estando el sector salud entre los grupos de mayor exposición (35\%)(5).

En abril de 2018, en un hospital de tercer nivel de Palma de Mallorca, se detectaron olores inhabituales en los laboratorios de microbiología (LAM) y análisis clínicos (LAC), y algunos profesionales del LAM manifestaron síntomas irritativos en mucosas (garganta, nariz y ojos), síntomas inespecíficos digestivos (náuseas) y mareos, que les llevaron a acudir al Servicio de Salud Laboral (SSL) del hospital y solicitar asistencia sanitaria. Según una primera información facilitada por la Dirección del Hospital, se sospechó que desde el 1 de marzo de 2018 se había producido un aumento en la incidencia de incapacidad temporal (IT) en los trabajadores de éstas áreas, pudiendo estar relacionada con la aparición de los malos olores.

El objetivo de este estudio fue confirmar o no la existencia de un brote y, de confirmarse, contribuir a la identificación de sus causas a fin de conseguir su control y prevención en el futuro.

\section{Métodos}

De acuerdo a los protocolos existentes en estas situaciones de posible brote epidémico ${ }^{(2,5-7)}$, y teniendo en cuenta las especificidades de la situación, se procedió a: a) establecer una definición de caso e identificar los casos, b) confirmar o no 
la existencia de un brote, c) caracterizar el brote organizando la información en términos de tiempo, lugar y persona (epidemiología descriptiva), d) Identificar las personas a riesgo de ser afectadas, e) desarrollar una hipótesis sobre el origen del brote, y f) recomendar medidas de prevención y control.

Para ello, se realizó en primer lugar un estudio cualitativo mediante la participación de los trabajadores con el objetivo específico de validar la definición operativa de caso, así como explorar las posibles explicaciones del brote de acuerdo a las percepciones de los trabajadores. En segundo lugar, se desarrolló un estudio cuantitativo para verificar la existencia del brote epidémico y proceder a su caracterización, organizando la información en términos de tiempo, lugar y persona, de acuerdo a la definición operativa establecida.

La parte cualitativa del estudio está basada en la técnica de grupos focales, que se llevó a cabo en las dependencias del Hospital, y se organizó en tres grupos focales con 10 participantes en cada uno: dos grupos focales con personas del LAM (grupos LAM1 y LAM2) y uno con personas del LAC (grupo LAC1). Todos ellos se condujeron con el formato de sesiones semiestructuradas y exploratorias dirigidas por los investigadores, y todas contaron con la presencia de uno de ellos cuya función consistió en operar la logística de la sesión, registrando toda la información que pudiera ser relevante. Las sesiones tuvieron una duración de entre 60 y 90 minutos cada una y se solicitó el consentimiento de los participantes para proceder a la grabación de la sesión. En todos los grupos focales se utilizó una misma guía de preguntas previamente elaborada que se estructuró en tres bloques temáticos: definición del problema (definición operativa de caso), posibles hipótesis sobre su origen y posibles soluciones desde la perspectiva de los trabajadores. La transcripción escrita de las intervenciones de los participantes se realizó para todos los grupos focales a partir de su grabación, manteniendo en todo momento el anonimato de los participantes. Los comentarios realizados se agruparon según provinieran de los participantes del LAM o del LAC, identificando aquellos que daban respuesta a los objetivos planteados, recabando la información relevante y elaborando un informe sobre las conclusiones.

Con todo esto, se validó la definición operativa de caso, concretándola de dos maneras diferentes y complementarias: 1) Caso de incapacidad temporal: toda persona trabajadora de los LAC y/o LAM que tuviera registrado un episodio de incapacidad temporal, fuera por contingencia común o profesional durante 2017 y 2018; y 2) Caso de visita al Servicio de Salud Laboral (SSL): toda persona trabajadora de los LAC y/o LAM que acudió a una visita médica relacionada con el brote y quedó registrado en al menos una ocasión en el SSL durante 2018.

La parte cuantitativa del estudio se elaboró a partir de la información facilitada por la Dirección del Hospital, construyéndose una base de datos anonimizada y específica para este estudio con las variables de interés (sexo, edad, categoría profesional, tipo de contrato y antigüedad en la empresa) de todos los trabajadores de los LAM y LAC, siendo posible diferenciar los episodios de IT tanto por contingencias comunes (ITcc) como por contingencias profesionales (ITcP) de un mismo trabajador durante el periodo de observación y las visitas realizadas al SSL por motivos 
de salud. Por razones de confidencialidad, los episodios de IT no disponían de diagnóstico médico, y en este estudio se contabilizaron y analizaron solamente los nuevos episodios de ITcc e ITcp de las personas trabajadoras en cada semana durante todo el periodo de observación, al objeto de contabilizar exclusivamente los episodios semanales incidentes.

El periodo de observación fueron los años 2017 y 2018, para poder establecer comparaciones semanales, cuando fuera posible, entre el periodo de brote (2018) y el año anterior (2017). Por este motivo, se consideró población de estudio todas las personas trabajadoras de los LAC y LAM durante los años 2017 y 2018.

El análisis se basó en una descripción de la población de estudio según las variables género (hombre; mujer), edad (18-35 años; 36-50 años; >50 años), categoría profesional (técnicos; administrativos; enfermeros; facultativos), tipo de contrato (fijo; temporal) y antigüedad en la empresa ( $\leq 5$ años, 6-10 años; >10 años). Todas ellas se describieron por medio de su frecuencia (n) y porcentaje (\%). Asimismo, se calculó la incidencia de casos de IT y de visitas al SSL en 2018 en ambos laboratorios, según variables sociodemográficas, evaluando mediante la prueba Chi-Cuadrado de Pearson $\left(\chi^{2}\right)$ la significación estadística de las diferencias encontradas dentro de las categorías de las diferentes variables. Se consideró que las diferencias fueron estadísticamente significativas cuando el p-valor $<0,05$.

Finalmente, se representó gráficamente el número de casos semanales, tanto de IT (2017 y 2018) como de visitas al SSL (2018) por separado para los LAM y LAC.

\section{Resultados}

\section{Estudio cualitativo}

Las sesiones de los grupos focales del LAM presentaron grandes similitudes con relación a la caracterización de los olores, los efectos sobre la salud y la intensidad de los mismos, apreciándose una diferencia clara con el grupo focal del LAC, en el que los comentarios fueron más matizados. La mayoría de los trabajadores del LAM coincidieron en que las molestias se iniciaron a mediados de 2016. Destacaba que los síntomas se presentaron predominantemente en el colectivo de técnicos del laboratorio y mejoraban en los periodos de descanso (fines de semana, vacaciones o periodos de IT). En todos los grupos focales se tenía la percepción de que el SSL del hospital no había dado una respuesta adecuada al problema. En la Tabla 1 se presenta un resumen de los comentarios realizados por las personas participantes, separando los realizados por los trabajadores del LAM de los realizados por los del LAC.

Los participantes de los tres grupos focales definieron el problema en base a dos percepciones principales: "estar inhalando alguna sustancia en su puesto de trabajo que no deberían estar inhalando" y "estar sufriendo efectos sobre la salud a consecuencia de la presencia de algún agente no habitual en su puesto de trabajo". Todos coincidieron en que no existía un patrón estacional ni de días concretos 
en la aparición de los olores y definieron tres tipos de olor: olor a formol (solo en los primeros días), olor cítrico (presente aún en el momento de las entrevistas) y, ocasionalmente, olor a basura o podredumbre.

Tabla1: Frases destacadas del estudio cualitativo en los Grupos Focales.

\section{Transcripción}

\section{Principales comentarios de los GF del Laboratorio de Microbiología}

"Primero era formol, luego parece que cambiaron el formol por el citrus, y hemos olido como a basura" (...) "Como cuando pasa el camión de la basura y pasas por detrás"

"Nos parece que lunes y viernes huele especialmente. Pero durante un año siempre se ha olido, unos días más otros menos"

"Independientemente de los olores, que son molestos, es lo otro, ese tipo de aerosol que notamos y que respiramos y que nos irrita tanto que nos da tantos problemas."

"Esta es la impresión que tenemos la mayoría, que nos cae por encima"

"Hay veces que hemos salido todos del laboratorio... y hemos tenido que parar, al menos un rato." "Abrir las ventanas, a ver si se cambiaba el aire y volver al trabajo"

"Hay un cuarto, que se supone que es estanco, que tiene un aire acondicionado propio, que se supone que no está comunicado y huele a citrus."

"Yo puedo decir que el estado anímico en micro, con tanta baja y lo que tardaban en sustituirnos... el estrés era importante"

\section{Principales comentarios de los GF del Laboratorio de Análisis Clínicos}

"Tenemos la percepción de que hay algo que inhalamos que no deberíamos inhalar"

"(...) en un espacio cerrado en el que hay un sistema de ventilación es algo o que se volatiliza o que es volátil y que no proviene del entorno cercano"

"La preocupación no es lo que huelo, es lo que no huelo."

En resumen, y preguntados por sus hipótesis sobre el origen del problema, las más repetidas fueron: 1) se trataba de compuestos volátiles que procedían de otros laboratorios $u$ otras partes del hospital ya que no eran olores habituales del propio LAM; 2) los olores llegaban al LAM a través de las conducciones de aire porque parecían salir de las rejillas de impulsión y extracción de aire; 3) no se estaban empleando reactivos nuevos, ni máquinas diferentes ni se habían producido cambios de procedimiento, por lo que el origen debía estar en otra parte del hospital; y 4) no se apreciaba la existencia de un posible conflicto en las relaciones interpersonales entre los trabajadores o entre estos y la empresa que pudiera estar influyendo en el brote que se estaba analizando.

A todas las personas participantes se les planteó la posibilidad de proponer soluciones y, aunque no se tenía una idea clara, muchos participantes coincidían en proponer las siguientes: 1) revisar exhaustivamente, por alguna empresa independiente, las conducciones de los circuitos de aire que constaban en los planos del Hospital y verificar que coincidían con la realidad de las instalaciones, especialmente las de los laboratorios; 2) identificar las modificaciones estructurales realizadas en los dos últimos años y comprobar si se habían realizado correctamente; 3) identificar los compuestos volátiles presentes en el ambiente, cuantificarlos e 
identificar dónde se utilizan, revisando los procedimientos de su uso y los circuitos de evacuación; y 4) si no era posible encontrar la causa, plantear la posibilidad de cambiar la ubicación actual de los laboratorios.

\section{Estudio cuantitativo}

La población de las unidades afectadas en ambos laboratorios en 2018 (Tabla 2) fue mayoritariamente mujeres (69,5\% LAM y 71,8\% LAC), entre 36 y 50 años (43,9\% y $41,7 \%$ ) y con categoría profesional de técnicos (63,4 y 60,2\%). La mayoría de trabajadores del LAM tenían un contrato temporal (57,3\%), mientras que para la mayoría de los del LAC su contrato era fijo $(67,0 \%)$. En el LAM la mayor parte de trabajadores tenía una antigüedad inferior a los 5 años (61,0\%), y en cambio en el LAC fue inferior a 5 años o superior a 10 años (43,7\% para ambos grupos).

Tabla 2: Descripción de los casos (primeras visitas e incapacidad temporal) e incidencia (por 100) en el Laboratorio de Análisis Clínicos y en el Laboratorio de Microbiología del Hospital, según variables demográficas y laborales.

\begin{tabular}{|c|c|c|c|c|c|c|c|c|c|c|c|c|c|c|c|}
\hline \multicolumn{8}{|c|}{ LABORATORIO DE MICROBIOLOGÍA } & \multicolumn{8}{|c|}{ LABORATORIO ANALISIS CLÍNICOS } \\
\hline \multicolumn{2}{|c|}{$\begin{array}{l}\text { Total traba- } \\
\text { jadores }\end{array}$} & \multicolumn{3}{|c|}{$\begin{array}{l}\text { Primeras visitas } \\
\text { Salud Laboral }\end{array}$} & \multicolumn{3}{|c|}{$\begin{array}{l}\text { Primeros episo- } \\
\text { dios Incapacidad } \\
\text { temporal }\end{array}$} & \multicolumn{2}{|c|}{$\begin{array}{l}\text { Total tra- } \\
\text { bajadores }\end{array}$} & \multicolumn{3}{|c|}{$\begin{array}{l}\text { Primeras visitas } \\
\text { Salud Laboral }\end{array}$} & \multicolumn{3}{|c|}{$\begin{array}{c}\text { Primeros episo- } \\
\text { dios Incapacidad } \\
\text { temporal }\end{array}$} \\
\hline$n$ & $\%$ & $\mathrm{n}$ & 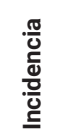 & $\begin{array}{l}\frac{\pi}{2} \\
\frac{*}{\pi} \\
\frac{\pi}{3} \\
\vdots\end{array}$ & $n$ & 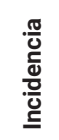 & 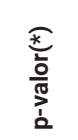 & $\mathbf{n}$ & $\%$ & $\mathbf{n}$ & 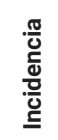 & 跑 & $\mathrm{n}$ & $\begin{array}{l}\frac{\pi}{0} \\
\frac{\mathrm{C}}{0} \\
\frac{0}{0} \\
\underline{\underline{0}}\end{array}$ & 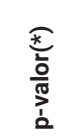 \\
\hline 25 & 30,5 & 6 & 24,0 & 0,205 & 8 & 32,0 & 0,387 & 29 & 28,2 & 0 & 0,0 & 0,271 & 5 & 17,2 & 0,162 \\
\hline 57 & 69,5 & 20 & 35,1 & & 24 & 42,1 & & 74 & 71,8 & 3 & 4,1 & & 24 & 32,4 & \\
\hline 24 & 28,0 & 4 & 16,7 & 0,394 & 7 & 29,2 & 0,671 & 25 & 24,3 & 0 & 0,0 & 0,546 & 4 & 16,0 & 0,248 \\
\hline 45 & 43,9 & 11 & 24,4 & & 16 & 35,6 & & 43 & 41,7 & 2 & 4,7 & & 15 & 34,9 & \\
\hline 34 & 28,0 & 11 & 32,4 & & 9 & 26,5 & & 35 & 34,0 & 1 & 2,9 & & 10 & 28,6 & \\
\hline 69 & 63,4 & 21 & 30,4 & 0,020 & 26 & 37,7 & 0.272 & 62 & 60,2 & 1 & 1,6 & 0,613 & 24 & 38,7 & 0,002 \\
\hline 10 & 12,2 & 1 & 10,0 & & 2 & 20,0 & & 13 & 12,6 & 1 & 7,7 & & 2 & 15,4 & \\
\hline 2 & 2,4 & 2 & 100 & & 1 & 50,0 & & 6 & 5,8 & 0 & 0,0 & & 3 & 50,0 & \\
\hline 18 & 22,0 & 2 & 11,1 & & 3 & 16,7 & & 22 & 21,4 & 1 & 4,5 & & 0 & 0,0 & \\
\hline 35 & 42,7 & 19 & 54,3 & $<0,001$ & 19 & 54,3 & 0,014 & 69 & 67,0 & 2 & 2,9 & 0,981 & 22 & 31,9 & 0,231 \\
\hline 47 & 57,3 & 7 & 14,9 & & 13 & 27,7 & & 34 & 33,0 & 1 & 2,9 & & 7 & 20,6 & \\
\hline 50 & 61,0 & 10 & 20,0 & $<0,001$ & 18 & 36,0 & 0,387 & 45 & 43,7 & 1 & 2,2 & 0,657 & 11 & 24,4 & 0,762 \\
\hline 5 & 6,1 & 0 & 0,0 & & 1 & 20,0 & & 13 & 12,6 & 0 & 0,0 & & 4 & 30,8 & \\
\hline 27 & 32,9 & 16 & 59,3 & & 13 & 48,1 & & 45 & 43,7 & 2 & 4,4 & & 14 & 31,1 & \\
\hline 82 & 100 & 26 & 31,7 & & 32 & 39,0 & & 103 & 100 & 3 & 2,9 & & 29 & 28,2 & \\
\hline
\end{tabular}

${ }^{*}$ ) P-valor calculado mediante el test Chi Cuadrado de Pearson $\left(\chi^{2}\right)$, considerando que existe significación estadística cuando p<0,05 
En el LAM la incidencia acumulada de visitas al SSL en 2018 fue del 31,7\% ( $n=26)$ y la de los episodios de IT fue del 39,0\% ( $n=32)$, no encontrando diferencias significativas según las variables de sexo y edad. Se encontraron diferencias significativas en la incidencia de visitas al SSL en las categorías de las variables tipo de contrato $(p<0,001)$ y antigüedad $(p<0,001)$ y en la incidencia de episodios de IT en las categorías de la variable tipo de contrato $(p=0,014)$.

En el LAC la incidencia acumulada de visitas al SSL fue del 2,9\% (n=3) y la de episodios de IT fue del $28,2 \%(n=29)$. No se encontraron diferencias significativas en la incidencia de visitas al SSL entre las categorías de ninguna de las variables analizadas. En la incidencia de IT únicamente se encontraron diferencias significativas dentro de los subgrupos de la variable categoría profesional $(p=0,002)$.

En el análisis temporal se observó que los casos incidentes semanales de IT del LAM en 2018, en comparación con el año 2017, presentan un incremento de entre uno y cuatro casos adicionales entre las semanas 21-39 del 2018 (Figura 1). Por otra parte, los casos semanales de IT del LAC presentaron un ligero incremento entre las semanas 19-21 de 2018 respecto de 2017 (1-2 casos semanales adicionales) (Figura 2).

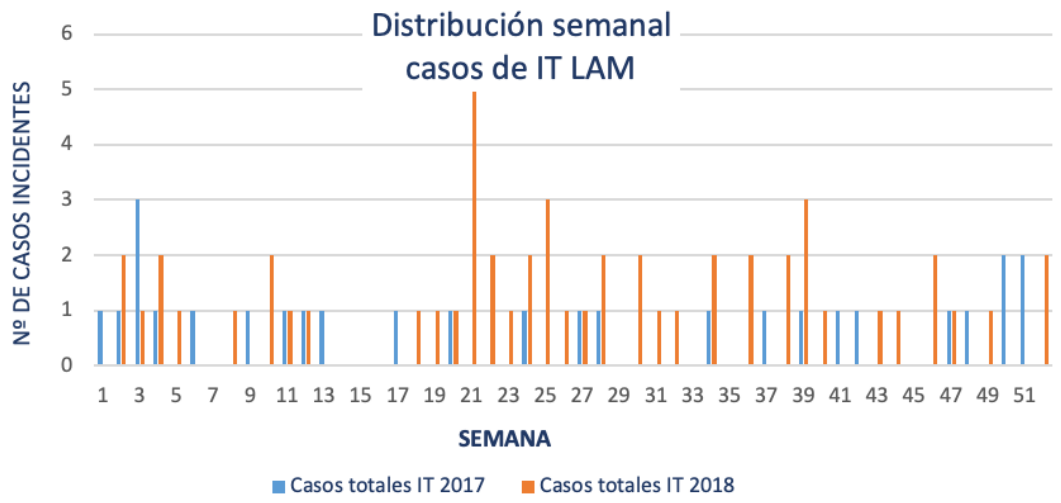

Figura 1. Distribución semanal de casos incidentes de incapacidad temporal (IT) del laboratorio de microbiología (LAM) años 2017 y 2018.

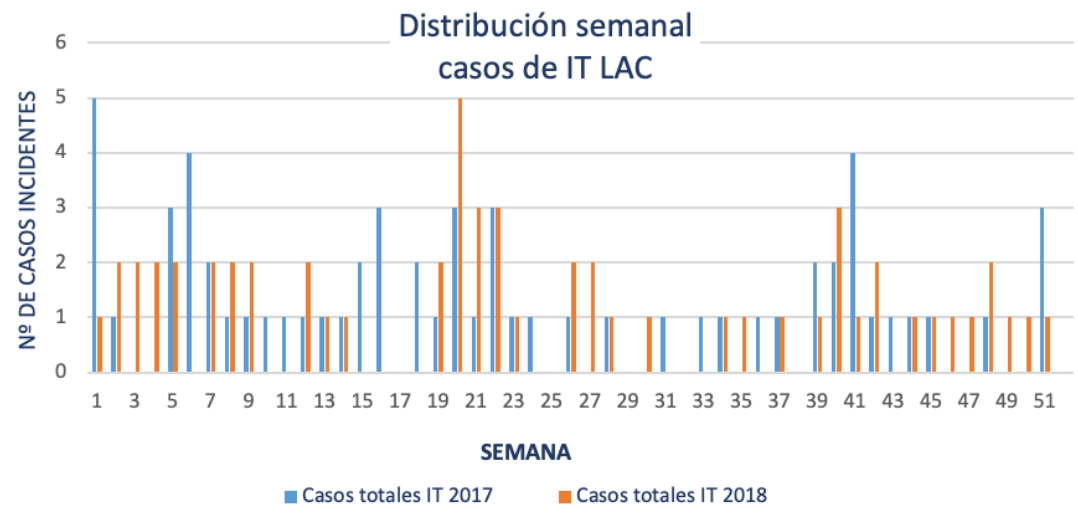

Figura 2. Distribución semanal de casos incidentes de incapacidad temporal (IT) del laboratorio de microbiología (LAC) años 2017 y 2018. 
En cuanto al número de visitas al SSL a lo largo de 2018, se observó un incremento en los trabajadores del LAM a partir de la semana $16(n=15)$, alcanzando un pico máximo en la semana $19(\mathrm{n}=30)$, manteniéndose un número de visitas variable hasta la semana 39. Sin embargo, los trabajadores del LAC apenas realizaron visitas al SSL, a excepción del periodo comprendido entre las semanas 16-22 en que se observaron 1-2 visitas semanales (Figura 3).

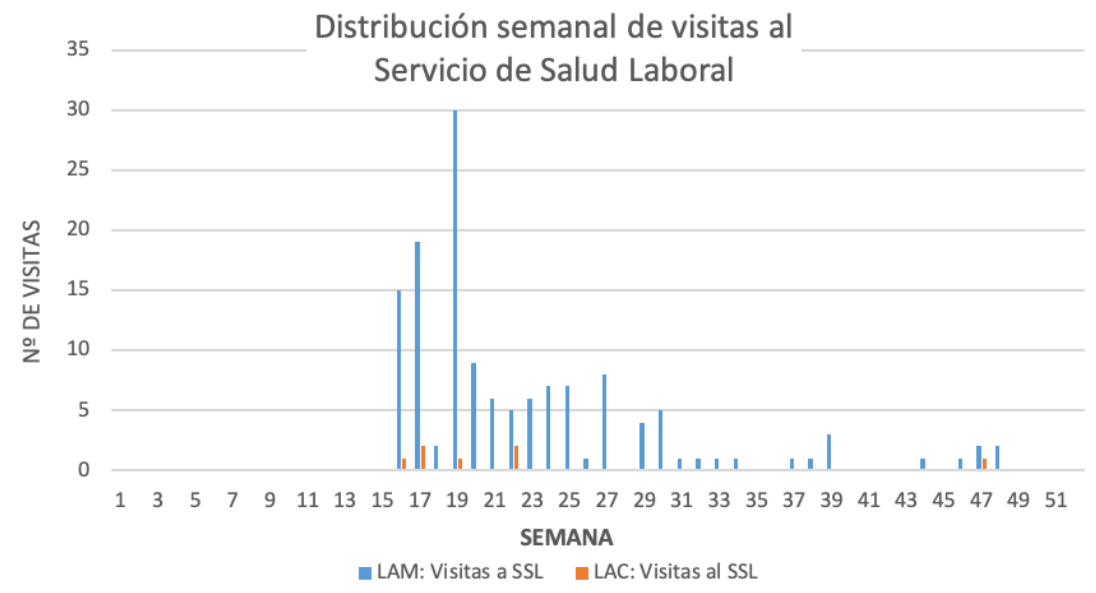

Figura 3. Distribución semanal de casos de visitas al Servicio de Salud Laboral (SSL) del laboratorio de microbiología (LAM) y del laboratorio de análisis clínicos (LAC) año 2018.

En la Figura 4 y Figura 5 se muestran las curvas epidémicas del LAM y del LAC, en las que se representa gráficamente la evolución del número de visitas al SSL y de casos incidentes de IT a lo largo de 2018. Cabe destacar que en el LAM, el incremento de visitas al SSL precede en varias semanas al incremento de los casos incidentes de IT.

En el análisis espacial se observó que en el LAM el mayor número de casos de IT $(n=30)$ y de visitas al SSL $(n=150)$ se produjeron en una de las áreas de este laboratorio, denominada laboratorio de bacteriología general y parasitología.

\section{CURVAS EPIDÉMICAS LAM 2018}

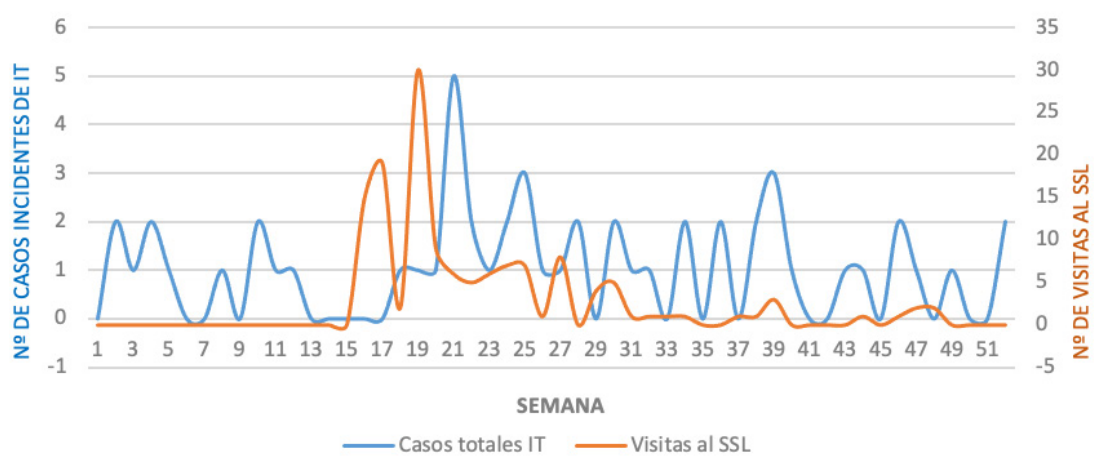

Figura 4. Curvas epidémicas del LAM. Distribución semanal de los casos de incapacidad temporal (IT) y de visitas al Servicio de Salud Laboral (SSL) del laboratorio de microbiología, 2018. 


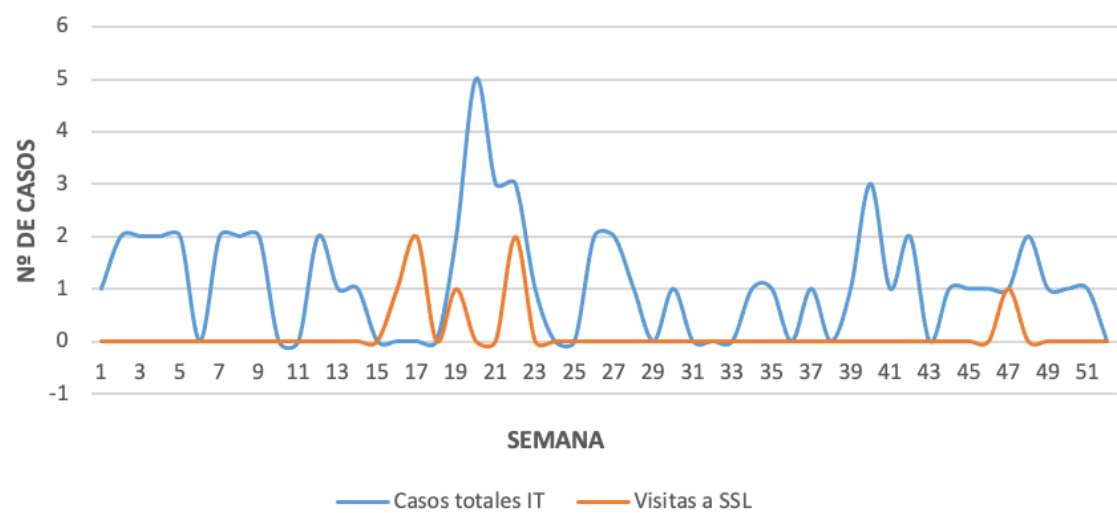

Figura 5. Curvas epidémicas del LAC. Distribución semanal de los casos de incapacidad temporal (IT) y de visitas al Servicio de Salud Laboral (SSL) del laboratorio de análisis clínicos, 2018.

\section{Discusión}

Los resultados muestran con claridad que el número de episodios de IT que se presentaron en el LAM entre las semanas 16 y 39 excedió en cuatro veces el número de casos esperados para esta área tomando como referencia el mismo periodo del año anterior. Un resultado que se confirma con las visitas al SSL, en base a la comparación con las semanas anteriores a la semana 16, pues no se dispuso de datos respecto de las visitas al SSL de 2017. También, se observó un exceso de casos entre las semanas 16 y 39 frente a las semanas 1 y 15 . En ambas series, el exceso de casos observados se concentró principalmente en el área de bacteriología general y parasitología del LAM. Por el contrario, el número de episodios de IT observados en el LAC entre las semanas 16 y 39 no excedió del número de casos esperados para esta área. Por ello, y con la información disponible, no se puede afirmar que haya existido un brote de las mismas características en este mismo periodo en el LAC.

A pesar de disponer solo de un año de comparación, normalmente se utilizan cinco años para calcular un índice endemo-epidémico ${ }^{(8)}$, estos resultados confirman la aparición de un brote epidémico, en cuanto que se observó un exceso de casos acumulados localizados fundamentalmente entre las semanas 16 y 39 en las áreas de bacteriología general y parasitología del LAM. Además, el hecho de que la curva epidémica construida en base a los registros de las visitas realizadas al SSL se presentara con unas semanas de antelación respecto de la curva construida en base a los episodios de IT, pues la visita al médico del SSL antecede a la visita a su médico de cabecera para solicitar la baja por IT, y que esto coincidiera en las mismas áreas del LAM, apoyó la hipótesis de la existencia de un brote epidémico principalmente en este laboratorio. 
Es importante considerar que la IT es un fenómeno altamente estacional, y que el pico de casos de IT en el presente estudio se produce entre las semanas 21-39 del 2018, dentro de las semanas de menor incidencia de IT a nivel general, lo cual apoyaría nuestra hipótesis de que el exceso de casos de IT en este periodo podrían atribuirse al brote detectado en el LAM.

Asimismo, y de acuerdo con la información aportada por las personas trabajadoras en los grupos focales, los principales síntomas descritos (irritación de garganta, nariz y ojos, náuseas y mareos) se asociaban a olores inhabituales y desagradables que fueron especialmente intensos en aquellas semanas, si bien habían continuado con posterioridad a aquellas semanas identificadas en el estudio cuantitativo. Igualmente, estas molestias y malos olores afectaron principalmente a los trabajadores del LAM.

Sin embargo, no se encontró una fuente de exposición, única o múltiple, ni sustancias específicas que explicaran el brote detectado. Las diversas inspecciones técnicas y los estudios ambientales a los que tuvimos acceso, realizados a petición de la Dirección del Hospital y llevados a cabo por Sociedad Concesionaria Hospital (responsable del mantenimiento de las instalaciones) y por los Servicios Científico-técnicos de la Universitat de les Illes Balears, no encontraron alteraciones en el funcionamiento de las instalaciones de ventilación, ni sustancias tóxicas por encima de su valor límite ambiental (VLA). También se llevó a cabo un estudio de los procesos de renovación del aire y medición de partículas, concluyendo que el número de partículas en suspensión permitía confirmar que la calidad del aire era buena. Asimismo, el propio Servicio de Salud Laboral del Hospital solicitó la realización de determinaciones ambientales de formaldehido en el LAM, encontrándose igualmente valores por debajo de los VLA establecidos. Por último, señalar que como consecuencia de la situación creada, se adoptaron medidas de mejora en las instalaciones, como la sustitución de filtros de carbono activo y el sellado preventivo de conducciones, de la preinstalación de vacío y gases medicinales, de imperfectos, holguras y huecos, sin que desaparecieran del todo los olores y molestias de acuerdo a lo expresado en los grupos focales, aunque el número de casos de IT y de visitas al SSL no volvieron a frecuencias similares a las semanas del brote.

Como todo estudio, el presente tiene algunas limitaciones. La más importante es no haber dispuesto del diagnóstico de los casos de IT, por lo que se asumió que todos los casos fueron debidos a la sintomatología referida por los trabajadores durante el periodo de brote. Asimismo, el presente estudio tiene la limitación de la posible existencia de cierta subjetividad que puede estar detrás de las visitas al SSL, y en menor medida, al médico de cabecera. El hecho de que se trate de síntomas inespecíficos, sin un diagnóstico claro puede estar sesgando de algún modo la atribución de algunos casos al periodo de brote. Igualmente, es remarcable el periodo de tiempo transcurrido desde la aparición del brote hasta su estudio ya que, cuando aparece un brote, la investigación debe ocurrir inmediatamente después de la notificación para no perder datos importantes para el análisis ${ }^{(8,9)}$. Por último, destacamos como limitación el no disponer de un periodo de tiempo previo 
de control más amplio. No obstante, a pesar de estas limitaciones, cabe destacar que este estudio se basó en la mejor información disponible, procedente tanto de un estudio cualitativo, que facilitó la participación de las personas que trabajan, y otro cuantitativo basado en dos fuentes de datos diferentes que reforzaron los resultados.

En conclusión, la investigación epidemiológica es necesaria, salvando las distancias, tanto en las pandemias por riesgos biológicos como en el estudio de brotes epidémicos (más modestos) por riesgos químicos. Este estudio epidemiológico ha mostrado que ha existido un brote, sin que se identificara un foco de exposición o sustancia que lo explicase. Por ello es recomendable mejorar los sistemas de vigilancia epidemiológica, basado en las dos fuentes utilizadas en este estudio, focalizando en aquellos casos que sean coherentes con el cuadro de síntomas identificado en la fase cualitativa, lo que permitirá monitorizar de manera continua cualquier cambio en la tendencia, así como localizar el lugar específico del brote. Ello facilitaría una actuación preventiva rápida, así como analizar las posibles causas del mismo. Asimismo, sería recomendable que además de las actuaciones llevadas a cabo por la Dirección y el Servicio de Prevención se verificara exhaustivamente el cumplimiento de las recomendaciones realizadas por la Sociedad Española de Enfermedades Infecciosas y Microbiología Clínica (SEIMC) relativas a los Procedimientos en Microbiología Clínica ${ }^{(10)}$.

\section{Agradecimientos}

Este estudio fue realizado a petición de la Dirección del Hospital mediante un contrato con CiSAL-UPF/IMIM, siguiendo una indicación de la Inspección de Trabajo de Les Illes Balears. Los investigadores tuvieron total autonomía para realizar las visitas y entrevistas que consideraron oportunas, y pudieron acceder a la información disponible relacionada con la investigación, respetando siempre la confidencialidad de los datos y el derecho a la privacidad de las personas. Los Comités de Empresa y de Seguridad y Salud en el Trabajo del Hospital estuvieron informados y asistieron a una sesión especial en que se les presentaron los resultados de la investigación.

\section{Bibliografía}

1. World Health Organization. Environmental health in emergencies [Internet]. Geneva: WHO; 2020 [citado 4 abril 2020]. Disponible en: https://www.who.int/environmental_health_emergencies/disease_outbreaks/en/

2. Horcajada JP, Padilla B. Endemia y epidemia. Investigación de un brote epidémico nosocomial. Enferm Infecc Microbiol Clin. 2013;31:181-186.

3. Maltseva A, Serra C, Kogevinas M. Cancer risk among workers of a secondary aluminium smelter. Occup Med (Lond). 2016;66:412-4. 
4. Instituto Nacional de Seguridad e Higiene en el Trabajo. Encuesta Nacional de Condiciones de Trabajo. $20156^{\text {a }}$ EWCS - España [Internet]. Madrid: INSHT; 2017 [citado 4 abril 2020] pp. 5-123. Disponible en: https://www.insst.es/documents/94886/96082/Encuesta+Nacional+de+Condiciones+de+Trabajo+6\%C2\%AA+EWCS/abd69b73-23ed-4c7f-bf8f-6b46f1998b45

5. Organización Panamericana de la Salud (OPS). Guía de Sistemas de Vigilancia de las Enfermedades Transmitidas por Alimentos (VETA) y la Investigación de Brotes. Capítulo III- Investigación de brotes en las personas [Internet]. Washington, D.C.: OPS; 2020 [citado 4 abril 2020]. Disponible en: https://www.paho.org/hq/index.php?option=com_content\&view=article\&id=10543:2015-capitulo-iii-investigacion-brotes-personas\&ltemid=41414\&lang=es

6. Celentano D, Szklo M. Gordis Epidemiología. 5a ed. Madrid: Elsevier; 2019.

7. Argimon-Pallas JM, Jimenez-Vila J. Métodos de investigación clínica y epidemiològica. 5a ed. Madrid: Elsevier; 2019.

8. González Ayala SE. Introducción a la Vigilancia Sanitaria. Análisis Epidemiológico [Internet]. Washington, D.C.: Organización Panamericana de la Salud (OPS); 2020 [citado 4 abril 2020]. Disponible en: https://www.paho.org/arg/publicaciones/publicaciones\%20virtuales/libroETAs/modulo6/modulo6d.html

9. Lezáun M. Intoxicaciones de origen laboral. Anales Sis San Navarra. 2003;26:265273.

10. Pérez Sáenz J (coordinador), Gómez García de la Pedrosa E, Leiva León J, Pérez Sáenz J, Rojo Molinero E. Procedimientos en Microbiología Clínica. Recomendaciones de la Sociedad Española de Enfermedades Infecciosas y Microbiología Clínica [Internet]. Madrid: Cercenado Mansilla E, Cantón Moreno R (ed); 2014 [citado 4 abril 2020] pp. 7-47. Disponible en: https://www.seimc.org/contenidos/ documentoscientificos/procedimientosmicrobiologia/seimc-procedimientomicrobiologia10a.pdf 